\title{
Dynamic changes and multiplication rate of white blood cell count may direct the timing of cytoreduction chemotherapy during induction treatment in newly diagnosed acute promyelocytic leukemia with low-intermediate risk
}

\author{
JINGJING WEN*, FANG XU*, QIAOLIN ZHOU, HONG HU, YIPING LIU, \\ JING SU, YAZHANG, WEN QU and LIN SHI

\begin{abstract}
Department of Hematology, Mianyang Central Hospital, School of Medicine, University of Electronic Science and Technology of China, Mianyang, Sichuan 621000, P.R. China
\end{abstract}

Received December 20, 2020; Accepted March 12, 2021

DOI: $10.3892 / \mathrm{mco} .2021 .2274$

\begin{abstract}
In order to explore the optimal timing for initiating cytoreduction chemotherapy following all-trans retinoic acid plus arsenic trioxide administration, 58 newly diagnosed patients with acute promyelocytic leukemia (APL) with low-intermediate mortality risk were retrospectively analyzed. During induction treatment, white blood cell (WBC) count $>4 \times 10^{9} / 1$ and multiplication rate of $\mathrm{WBC}<3$ days were defined as rapid WBC multiplication. Patients were divided into two groups: With or without rapid WBC multiplication. Comparison between the two groups revealed that the incidence of differentiation syndrome (DS) $(48.1 \%$ vs. 6.5\%; $\mathrm{P}<0.001)$, grade $3-4$ bleeding $(34.8 \%$ vs. $6.5 \% ; \mathrm{P}=0.022)$ and peak WBC count $\left(30.4 \pm 20.0 \times 10^{9} / 1\right.$ vs. $8.67 \pm 5.4 \times 10^{9} / 1$; $\mathrm{P}<0.001)$ were significantly higher in the group with rapid WBC multiplication compared with in the group without rapid WBC multiplication. No significant differences were observed in bone marrow depression, infection, complete remission (CR) rate, time to achieve CR and early mortality rate between the two groups. Multivariate analysis revealed that WBC
\end{abstract}

Correspondence to: Dr Fang Xu, Department of Hematology, Mianyang Central Hospital, School of Medicine, University of Electronic Science and Technology of China, 12 Changjia Alley, Jingzhong Street, Fucheng, Mianyang, Sichuan 621000, P.R. China E-mail: 147377807@qq.com

${ }^{*}$ Contributed equally

Abbreviations: APL, acute promyelocytic leukemia; ATRA, all-trans retinoic acid; ATO, arsenic trioxide; NCCN, National Comprehensive Cancer Network; WBC, white blood cell; DS, differentiation syndrome; CR, complete remission; PLT, platelet; ECOG, Eastern Cooperative Oncology Group

Key words: APL, WBC, multiplication rate, low-intermediate risk, induction treatment, initiation time, cytoreduction chemotherapy count at chemotherapy initiation was an independent risk factor for the occurrence of DS $(\mathrm{P}=0.040)$. Peak WBC count and rapid $\mathrm{WBC}$ multiplication were significantly associated with grade $3-4$ bleeding ( $\mathrm{P}=0.019$ and $\mathrm{P}=0.002$, respectively). Hence, WBC count at chemotherapy initiation along with its multiplication rate may direct the timing of cytoreduction chemotherapy during induction treatment in newly diagnosed APL with low-intermediate risk.

\section{Introduction}

All-trans retinoic acid (ATRA) and arsenic trioxide (ATO) are two basic drugs for induction treatment for newly diagnosed acute promyelocytic leukemia (APL). Based on numerous clinical trials $(1,2)$, the National Comprehensive Cancer Network (NCCN) guideline recommends ATRA combined with ATO as the standard induction treatment for de novo APL with low risk [white blood cell (WBC) count $<10 \times 10^{9} / 1$ (3). Only if there are any contraindications to arsenic, ATRA combined with idarubicin could be considered (4-6). Chinese APL guideline also recommends ATRA combined with ATO as basic treatment for induction therapy for de novo APL (7). Anthracyclines or other cytoreduction chemotherapy could be chosen if WBC count is $>10 \times 10^{9} / 1$. After administration of ATRA and ATO, WBC count will typically rise and may lead to differentiation syndrome (DS). Cytoreduction chemotherapy may partially prevent or control the risks of DS. However, no guidelines give specific suggestions about when and how to initiate cytoreduction chemotherapy. Our previous research indicated that it was better to initiate cytoreduction chemotherapy when WBC count was between $4 \times 10^{9} / 1$ and $15 \times 10^{9} / 1$ during induction treatment for APL with low-intermediate risk (8). It is difficult for clinicians to decide to use chemotherapy when WBC count is not very high. However, the WBC count occasionally multiplies and exceeds $15 \times 10^{9} / 1$ very rapidly before clinicians can make a decision. To assess the appropriate time to initiate cytoreduction therapy during induction treatment, static WBC count may not be universally satisfactory. Hence, this study was designed to explore the relationship between the 
multiplication rate of WBC count and clinical effects during ATRA plus ATO-based induction treatment for patients with low-intermediate risk APL.

\section{Patients and methods}

Patients. Between January 2015 and December 2020, a total of 92 newly diagnosed patients were admitted to our hematology department. Of these, 60 patients were categorized into the low-intermediate risk group, and the remaining patients were categorized into the high risk group, based on Sanz's risk stratification model for survival prediction (9). All 58 patients ( 34 males and 24 females), with a median age of 34 years (range 17-71 years)] with low-intermediate risk, who had complete clinical data, were retrospectively enrolled in this study. Disease diagnosis was confirmed by bone marrow aspiration, chromosome karyotyping analysis, fluorescence in situ hybridization analysis and polymerase chain reaction. Informed consent prior to and regarding the treatment protocol was obtained from all patients analyzed in the present study.

Treatments. When a diagnosis of APL was suspected, ATRA $\left(20 \mathrm{mg} / \mathrm{m}^{2} /\right.$ day $)$ was administered at the earliest, until complete remission (CR) was achieved. All 58 patients received ATRA and ATO-based induction treatment. If the WBC count increased, cytotoxic agents-based chemotherapy was initiated based on the clinician's decision in order to reduce leukemia cells and prevent risks of DS. A total of 19 cases received idarubicin $\left(8 \mathrm{mg} / \mathrm{m}^{2} /\right.$ day on days $\left.1-3\right)$, 32 cases received daunorubicin ( $45 \mathrm{mg} / \mathrm{m}^{2} /$ day on days $\left.1-3\right)$, two cases received daunorubicin ( $45 \mathrm{mg} / \mathrm{m}^{2} /$ day on days $\left.1-3\right)$ combined with cytarabine $\left(100 \mathrm{mg} / \mathrm{m}^{2} /\right.$ day on days $\left.1-7\right)$, and five cases received only hydroxyurea (2.0-3.0 g/day, adjusted according to blood cells). Blood product support was applied to maintain platelet (PLT) level $\geq 30 \times 10^{9} / 1$, hemoglobin level $\geq 70 \mathrm{~g} / \mathrm{l}$ and plasma fibrinogen level $\geq 1.5 \mathrm{~g} / \mathrm{l}$. All adverse events related to treatments, including bone marrow depression, infection and bleeding, were graded according to the National Cancer Institute Common Terminology Criteria for Adverse Events version 4.03 (10).

Definitions and groups. DS was diagnosed based on the incidence of at least two of the following clinical features: Unexplained fever, acute respiratory distress with interstitial pulmonary infiltrates, acute renal failure, weight gain $>5 \mathrm{~kg}$, unexplained hypotension, and pleuropericardial effusion (11). Prevention strategies included dexamethasone (10 mg q12h), and discontinuation of ATRA and ATO. All 58 patients were categorized according to Sanz's risk stratification score (9) as low risk (WBC count $<10.0 \times 10^{9} / 1$ and PLT $\geq 40.0 \times 10^{9} / 1$ at diagnosis) and intermediate risk (WBC count $<10 \times 10^{9} / 1$ and PLT $\left.<40.0 \times 10^{9} / 1\right)$. CR was defined as the presence of $<5 \%$ blast cells in bone marrow aspirates, PLT $>100 \times 10^{9} / 1$ and no juvenile cells in peripheral blood according to the criteria established by the US National Cancer Institute (12). Early mortality was defined as fatality during induction treatment from the first day of hospitalization. Eastern Cooperative Oncology Group (ECOG) performance status score (0-4 score) was applied to investigate the patient's physical status prior to chemotherapy (13). WBC count $>4 \times 10^{9} / 1$ and WBC multiplication time $<3$ days was defined as rapid WBC multiplication. Table I presents a comparison of the baseline clinical and laboratory parameters of the groups with rapid $\mathrm{WBC}$ multiplication and without rapid WBC multiplication.

Statistical analysis. Statistical analysis was performed with SPSS v.22.0 software (SPSS Inc.). All data were collected in December 2020. Clinical features are presented as percentages (\%) for categorical variables, and as mean values \pm standard deviation for normally distributed continuous variables. The $\chi^{2}$ test was used to analyze the significance of differences in the distribution of categorical variables between the patient subsets, and the SNK method and Bonferroni's correction were used to compare the two groups. The unpaired Student's t-test or Mann-Whitney U test was used to analyze the significance of differences in the distributions of continuous parametric variables and ranked variables. Multivariate analysis was performed using a binary logistic regression model. WBC count at diagnosis, WBC count at chemotherapy initiation, peak WBC count, and with or without rapid WBC multiplication were considered in the multivariate analysis to evaluate their effects on DS, early mortality and grade 3-4 bleeding. $\mathrm{P}<0.05$ was considered to indicate statistical significance.

\section{Results}

Comparisons of clinical effects between patients with and without rapid WBC multiplication. Comparison of the baseline features, clinical and laboratory parameters is shown in Table I. No significant differences were found in age, gender and ECOG score between the two groups. WBC count at diagnosis was slightly higher in the group with rapid WBC multiplication $\left(2.14 \pm 1.66 \times 10^{9} / 1\right.$ vs. $3.82 \pm 2.98 \times 10^{9} / 1$, $\mathrm{P}=0.013)$. There was no difference between the two groups in Sanz's risk stratification $(\mathrm{P}=0.121)$. Biochemical tests showed no differences in uric acid, albumin and creatine levels in patients with or without rapid WBC multiplication. Coagulation functions including activated partial thromboplastin, prothrombin time, fibrinogen and D-dimer level were similar in the two groups.

As depicted in Table II, the incidence of DS was higher (48.1 vs. $6.5 \%, \mathrm{P}=0.000$ ), grade $3-4$ bleeding was more frequent ( 34.8 vs. $6.5 \%, \mathrm{P}=0.022)$, and peak WBC count was higher $\left(30.4 \pm 20.0 \times 10^{9} / 1\right.$ vs. $\left.8.67 \pm 5.4 \times 10^{9} / 1, \mathrm{P}=0.000\right)$ in the rapid WBC multiplication group. There were no differences in bone marrow depression, infection, $\mathrm{CR}$ rate, time to achieve $\mathrm{CR}$ and early mortality rate between the two groups.

Multivariate associations. To further evaluate the effects of dynamic changes of WBC count during induction treatment on DS, early mortality and bleeding events, a binary logistic regression model was used. The results of multivariate analysis revealed that WBC count at chemotherapy initiation was an independent risk factor for the occurrence of DS $[\mathrm{P}=0.040$, odds ratio $(\mathrm{OR})=1.0216,95 \%$ confidence interval $(\mathrm{CI})=1.009-1.465]$. Peak WBC count and rapid WBC multiplication were significantly related to grade 3-4 bleeding $(\mathrm{P}=0.019, \mathrm{OR}=0.773,95 \% \mathrm{CI}=0.623-0.959 ; \mathrm{P}=0.002$, $\mathrm{OR}=99.567,95 \% \mathrm{CI}=5.738-1727.726)$. However, WBC count at 
Table I. Baseline demographic, clinical and laboratory characteristics of groups without ( $\mathrm{n}=31)$ or with $(\mathrm{n}=27)$ rapid WBC multiplication.

\begin{tabular}{|c|c|c|c|}
\hline Characteristics & Without rapid WBC multiplication & With rapid WBC multiplication & P-value \\
\hline Age, years & $34.7 \pm 14.2$ & $32.3 \pm 9.0$ & 0.425 \\
\hline $15-54$ & $28(90.3)$ & $27(100.0)$ & \\
\hline $55-70$ & $3(9.7)$ & $0(0.0)$ & \\
\hline Sex, male/female & $19 / 12$ & $15 / 12$ & 0.658 \\
\hline Sanz's risk stratification & & & 0.121 \\
\hline Low risk & $10(32.3)$ & $4(14.8)$ & \\
\hline Intermediate risk & $21(67.7)$ & $23(85.2)$ & \\
\hline ECOG score & & & 0.315 \\
\hline $1-2$ & $19(61.3)$ & $13(48.1)$ & \\
\hline $3-4$ & $12(38.7)$ & $14(51.9)$ & \\
\hline WBC count at diagnosis, $\times 10^{9} / 1$ & $2.14 \pm 1.66$ & $3.82 \pm 2.98$ & 0.013 \\
\hline Platelet count at diagnosis, $\mathrm{x} 10^{9} / 1$ & $37.5 \pm 39.3$ & $22.7 \pm 17.6$ & 0.067 \\
\hline LDH, IU/1 & $303.3 \pm 170.7$ & $333.0 \pm 185.0$ & 0.542 \\
\hline Uric acid, $\mu \mathrm{mol} / \mathrm{l}$ & $277.3 \pm 97.7$ & $297.4 \pm 92.6$ & 0.657 \\
\hline Albumin, g/l & $42.1 \pm 3.8$ & $43.4 \pm 8.12$ & 0.451 \\
\hline Creatine, $\mu \mathrm{mol} / 1$ & $67.6 \pm 21.3$ & $67.6 \pm 22.3$ & 0.993 \\
\hline Activated partial thromboplastin, sec & $27.0 \pm 3.119$ & $44.2 \pm 50.2$ & 0.093 \\
\hline Prothrombin time, sec & $13.3 \pm 1.54$ & $33.1 \pm 54.1$ & 0.074 \\
\hline Fibrinogen, $\mathrm{g} / \mathrm{l}$ & $1.51 \pm 0.86$ & $1.40 \pm 0.81$ & 0.608 \\
\hline D dimer, mg/l & $9.38 \pm 33.1$ & $1.73 \pm 1.33$ & 0.224 \\
\hline Cytotoxic agent distribution & & & 0.584 \\
\hline Idarubicin & $8(25.8)$ & $11(40.7)$ & \\
\hline Daunorubicin & $17(54.8)$ & $15(55.6)$ & \\
\hline DA & $1(3.2)$ & $1(3.7)$ & \\
\hline Hydroxyurea & $5(16.1)$ & $0(0.0)$ & \\
\hline
\end{tabular}

Values are provided as $\mathrm{n}(\%)$ or as the mean $\pm \mathrm{SD}$. ECOG, Eastern Cooperative Oncology Group; WBC, white blood cell; LDH, lactate dehydrogenase; DA, daunorubicin and cytarabine.

diagnosis, WBC count at chemotherapy initiation, peak WBC count and rapid WBC multiplication were not independent risk factors for early mortality (Table III).

\section{Discussion}

European Leukemia Net updated the recommendations for management of APL in 2019 (14). For non-high-risk APL patients (WBC $\leq 10 \times 10^{9} / 1$ ), either ATRA plus ATO without chemotherapy or standard ATRA-idarubicin therapy were recommended as induction treatment, which was consistent with NCCN guidelines (3). In both guidelines, when and how to use cytoreduction therapy during ATRA plus ATO-based induction treatment was not discussed. However, the risks of DS may increase with the increase of WBC count, which may lead to other serious side effects. Hence, a randomized clinical trial was designed to compare the efficacy of ATRA-ATO vs. ATRA-ATO plus chemotherapy in all-risk patients with APL (15), but the results have not yet been published.

Our previous study found that WBC count can be conveniently used to direct cytoreduction chemotherapy during induction treatment for low-intermediate risk APL. When
WBC count is between $4 \times 10^{9} / 1$ and $15 \times 10^{9} / 1$, cytotoxic drugs could be initiated to control leukocytosis (8). However, for patients with different types of WBC count multiplication rate, single $\mathrm{WBC}$ count may be inadequate to decide on cytoreduction therapy. For slow type of WBC count multiplication, cytoreduction therapy may be optional, even if WBC count increases to $>10 \times 10^{9} / 1$. However, patients whose WBC count doubles rapidly, cytoreduction therapy should be considered even if WBC count is $<10 \times 10^{9} / 1$ because of DS risk. Hence, this study attempted to establish the effects of WBC dynamic changes along with static WBC count on early adverse events including early death, DS, grade 3-4 infection and bleeding.

Since there were no similar studies to be referred, we defined rapid WBC multiplication as $\mathrm{WBC}$ count $>4 \times 10^{9} / 1$ and multiplication time $<3$ days. It was a grouping method based on clinical experience. Comparison results indicated that patients with rapid WBC multiplication rate had higher possibility of DS and grade 3-4 bleeding, which was consistent with significantly higher peak WBC count in patients with rapid WBC multiplication. However, these adverse effects did not increase the incidence of early death. Multivariate analysis 
Table II. Comparison of clinical effects in groups without $(n=31)$ or with $(n=27)$ rapid WBC multiplication.

\begin{tabular}{|c|c|c|c|}
\hline Effects & Without rapid WBC multiplication & With rapid WBC multiplication & P-value \\
\hline DS incidence & $2(6.5)$ & $13(48.1)$ & $<0.001$ \\
\hline Grade 3-4 bone marrow depression & $26(83.9)$ & $24(88.9)$ & 0.864 \\
\hline Grade 3-4 infection & $16(51.6)$ & $13(48.1)$ & 0.792 \\
\hline Grade $3-4$ bleeding & $2(6.5)$ & $8(34.8)$ & 0.022 \\
\hline Valley WBC count $\times 10^{9} / 1$ & $1.11 \pm 0.66$ & $1.18 \pm 0.66$ & 0.695 \\
\hline Peak WBC count $\times 10^{9} / 1$ & $8.67 \pm 5.4$ & $30.4 \pm 20.0$ & $<0.001$ \\
\hline $\mathrm{CR}$ rate & $28(90.3)$ & $22(81.5)$ & 0.554 \\
\hline Time to achieve CR, days & $32.4 \pm 13.2$ & $28.8 \pm 6.1$ & 0.247 \\
\hline Early mortality rate & $3(9.7)$ & $5(18.5)$ & 0.554 \\
\hline \multicolumn{4}{|l|}{ Mortality cause, n (\%) } \\
\hline Hemorrhage & $1(33.3)$ & $3(50.0)$ & NA \\
\hline DS & $1(33.3)$ & $1(16.7)$ & \\
\hline Infection & $0(0.0)$ & $1(16.7)$ & \\
\hline Other & $1(33.3)$ & $1(16.7)$ & \\
\hline
\end{tabular}

Values are provided as $\mathrm{n}(\%)$ or as the mean $\pm \mathrm{SD}$. WBC, white blood cell; DS, differentiation syndrome; CR, complete remission; NA, not applicable.

Table III. Multivariate analysis of WBC count at diagnosis and CT initiation, peak WBC count and rapid WBC multiplication.

\begin{tabular}{|c|c|c|c|c|c|c|c|}
\hline Dependent variable & Independent variables & $\mathrm{B}$ & $\begin{array}{l}\text { Standard } \\
\text { error }\end{array}$ & $\begin{array}{c}\text { Wald } \\
\text { coefficient }\end{array}$ & P-value & Odds ratio & $\begin{array}{c}95 \% \text { confidence } \\
\text { interval }\end{array}$ \\
\hline \multirow[t]{4}{*}{ Differentiation syndrome } & WBC count at diagnosis & 0.273 & 0.169 & 2.610 & 0.106 & 1.314 & $0.943-1.830$ \\
\hline & WBC count at CT initiation & 0.196 & 0.095 & 4.238 & 0.040 & 1.216 & $1.009-1.465$ \\
\hline & Peak WBC count & 0.016 & 0.023 & 0.489 & 0.485 & 1.016 & $0.971-1.064$ \\
\hline & Rapid WBC multiplication & -0.987 & 1.350 & 0.534 & 0.465 & 0.373 & $0.026-5.259$ \\
\hline \multirow[t]{4}{*}{ Early mortality } & WBC count at diagnosis & 0.059 & 0.161 & 0.132 & 0.716 & 1.060 & $0.773-1.454$ \\
\hline & WBC count at CT initiation & 0.019 & 0.043 & 0.198 & 0.656 & 1.020 & $0.936-1.110$ \\
\hline & Peak WBC count & 0.000 & 0.030 & 0.000 & 0.987 & 1.000 & $0.943-1.059$ \\
\hline & Rapid WBC multiplication & -0.622 & 1.094 & 0.323 & 0.570 & 0.537 & $0.063-4.586$ \\
\hline \multirow[t]{4}{*}{ Grade $3-4$ bleeding } & WBC count at diagnosis & -0.183 & 0.216 & 0.715 & 0.398 & 0.833 & $0.546-1.272$ \\
\hline & WBC count at CT initiation & 0.191 & 0.109 & 3.099 & 0.078 & 1.211 & $0.979-1.498$ \\
\hline & Peak WBC count & -0.258 & 0.110 & 5.462 & 0.019 & 0.773 & $0.623-0.959$ \\
\hline & Rapid WBC multiplication & 4.601 & 1.456 & 9.985 & 0.002 & 99.567 & $5.738-1727.726$ \\
\hline
\end{tabular}

WBC, white blood cell; CT, chemotherapy.

further proved that WBC count in chemotherapy was the most important factor for DS, and WBC multiplication rate was closely related to serious bleeding events.

The main limitation of this study was its retrospective design. In this study, baseline characteristics between the two groups were not statistically equal, especially WBC count at diagnosis. However, mean WBC count at diagnosis in both groups was less than normal level. Further multivariate analysis showed that the WBC count at diagnosis was not the most important factor for DS, early mortality and grade 3-4 bleeding, compared to other three factors. To some extent, multivariate analysis corrected the bias caused by differences in baseline characteristics. Different from previous research, this study collected patient's data from another hospital, and all patients were treated with ATRA plus ATO. However, we obtained similar result that WBC count at chemotherapy initiation was associated with DS.

Overall, WBC count at chemotherapy initiation along with WBC multiplication rate could direct the timing of cytoreduction chemotherapy during induction treatment in newly diagnosed acute promyelocytic leukemia with low-intermediate risk.

\section{Acknowledgements}

Not applicable. 


\section{Funding}

The study was funded by a research project from Mianyang Health Commission (grant no. 201914).

\section{Availability of data and materials}

The datasets used and/or analyzed during the current study are available from the corresponding author on reasonable request.

\section{Authors' contributions}

FX conceived and designed the study, and provided administrative support. FX, JW and QZ designed the concept of the article and wrote the manuscript. FX and JW analyzed and interpreted the data, and confirmed the authenticity of the data. JW, QZ, HH, YL, JS, YZ, WQ and LS designed the concept of the article and collected the data. All authors contributed to the provision of study materials or patients. All authors read and approved the final manuscript.

\section{Ethics approval and consent to participate}

Ethics approval was obtained from the Ethics Committee of Mianyang Central Hospital (Mianyang, China; approval no. S-2019-099). Written informed consent prior to and regarding the treatment protocol was obtained from all patients analyzed in the present study.

\section{Patient consent for publication}

Not applicable.

\section{Competing interests}

The authors declare that they have no competing interests.

\section{References}

1. Sanz MA, Montesinos P, Rayon C, Holowiecka A, de la Serna J, Milone G, de Lisa E, Brunet S, Rubio V, Ribera JM, et al: Risk-Adapted treatment of acute promyelocytic leukemia based on all-trans retinoic acid and anthracycline with addition of cytarabine in consolidation therapy for highrisk patients: Further improvements in treatment outcome. Blood 115: 5137-5146, 2010.

2. Lo-Coco F, GAvvisati G, Vignetti M, Thiede C, Orlando SM, Iacobelli S, Ferrara F, Fazi P, Cicconi L, Di Bona E, et al: Retinoic acid and arsenic trioxide for acute promyelocytic leukemia. N Engl J Med 369: 111-112, 2013.

3. Tallman MS, Wang ES and Altman JK: Acute Myeloid Leukemia, Version 2.2020, NCCN Clinical Practice Guidelines in Oncology, National Comprehensive Cancer Network.
4. Mandelli F, Diverio D, Avvisati G, Luciano A, Barbui T, Bernasconi C, Broccia G, Cerri R, Falda M, Fioritoni G, et al: Molecular remission in PML/RAR alpha-positive acute promyelocytic leukemia by combined all-trans retinoic acid and idarubicin (AIDA) therapy. Gruppo italiano-malattie ematologiche maligne dell'Adulto and associazione Italiana di ematologia ed oncologia pediatrica cooperative groups. Blood 90: 1014-1021, 1997.

5. Sanz MA, Martin G, Rayon C, Esteve J, González M, DíazMediavilla J, Bolufer P, Barragán E, Terol MJ, González JD, et al: A modified AIDA protocol with anthracycline-based consolidation results in high antileukemic efficacy and reduced toxicity in newly diagnosed PML/RARalpha-positive acute promyelocytic leukemia. PETHEMA group. Blood 94: 3015-3021, 1999.

6. Fenaux P, Le Deley MC, Castaigne S, Archimbaud E, Chomienne C, Link H, Guerci A, Duarte M, Daniel MT and Bowen D: Effect of all transretinoic acid in newly diagnosed acute promyelocytic leukemia. Results of a multicenter randomized trial. European APL 91 group. Blood 82: 3241-3249, 1993.

7. Zhixiang S, et al: Chinese guidelines for diagnosis and treatment of acute promyelocytic leukemia. Zhonghua Xue Ye Xue Za Zhi 37: 179-183, 2016

8. Xu F, Yin CX, Wang CL, Ding BJ, Zhong QX, Jiang XJ, Jiang L, Wang ZX and Meng FY: Influence of initiation time and white blood cell count on the efficacy of cytotoxic agents in acute promyelocytic leukemia during induction treatment. Biomed Rep 39: 227-232, 2018.

9. Sanz MA, Lo Coco F, Martin G, Avvisati G, Rayón C, Barbui T, Díaz-Mediavilla J, Fioritoni G, González JD, Liso V, et al: Definition of relapse risk and role of nonanthracycline drugs for consolidation in patients with acute promyelocytic leukemia: A joint study of the PETHEMA and GIMEMA cooperative groups. Blood 96: 1247-1253, 2000.

10. Common Terminology Criteria for Adverse Events, Version 4.03, DCTD,NCI, NIH,DHHS. 14 June 2010.

11. Frankel SR, Eardley A, Lauwers G, Weiss M and Warrell RP Jr: The 'retinoic acid syndrome' in acute promyelocytic leukemia. Ann Intern Med 117: 292-296, 1992.

12. Cheson BD, Bennett JM, Kopecky KJ, Büchner T, Willman CL, Estey EH, Schiffer CA, Doehner H, Tallman MS, Lister TA, et al: Revised recommendations of the international working group for diagnosis, standardization of response criteria, treatment outcomes, and reporting standards for therapeutic trials in acute myeloid leukemia. J Clin Oncol 21: 4642-4649, 2003.

13. Oken MM, Creech RH, Tormey DC, Horton J, Davis TE, McFadden ET and Carbone PP: Toxicity and response criteria of the eastern cooperative oncology group. Am J Clin Oncol 5: 649-655, 1982.

14. Sanz MA, Fenaux P, Tallman MS, Estey EH, Löwenberg B, Naoe T, Lengfelder E, Döhner H, Burnett AK, Chen SJ, et al: Management of acute promyelocytic leukemia: Updated recommendations from an expert panel of the European leukemiaNet Blood 133: 1630-1643, 2019.

15. Zhang X, Zhang H, Chen L, Wang M, Xi J, Liu X, Xie M, Li D, Gulati ES, Gong S and Wang H: Arsenic trioxide and all-trans retinoic acid(ATRA)treatment for acute promyelocytic leukemia in all risk groups: Study protocol for a randomized controlled trial. Trials 19: 476, 2018. 